\title{
Damage Tolerance of Naval Sandwich Panels
}

\author{
Dan Zenkert \\ Department of Aeronautical and Vehicle Engineering \\ Kungliga Tekniska Högskolan \\ SE-10044 Stockholm, Sweden
}

\begin{abstract}
This paper is a review of activities concerning various damage tolerance modelling and testing aspects of sandwich panels for typical Naval applications. It starts with a review of testing methods for primarily core materials and how to extract properties and data required for damage tolerance assessment. Next some typical damage types are defined and how they are modelled with the aim of predicting their effect on load bearing capacity. The paper then describes in brief how such models can used in the context of providing a systematic damage assessment scheme for composite sandwich ship structures.
\end{abstract}

\section{Introduction and background}

Sandwich panels have been used to construct hulls and superstructures of Naval ships for quite some time. These comprise of composite face sheets (glass or carbon) separated by a low density core, usually polymeric foam.

The main driver for using composite sandwich structures in ship hulls is otherwise low structural weight. Ships are often built in short or very short series, implying that using composites can be cost effective too. Other important features are the non-corrosive material that leads to much lower maintenance costs in the lifetime of the structure. Maintenance costs in the order of $15 \%$ of those of a similar steel ship have been reported. In Naval applications, a non-magnetic hull material is very advantageous and sometimes necessary. Other important features of composite sandwich hulls are low acoustic and thermal signatures. Through the development of various naval sandwich ships, one has also found that sandwich panels are inherently blast resistant compared to steel or single skin structures.

Historically, sandwich structures were first used in mine counter measure vessels, mainly designed for underwater blast protection UNDEX, such as the Landsort class depicted in Fig.1a. Having relatively low speed, these vessels became very much over-designed for normal sea loads and thus very damage tolerant under normal usage. However, ships with other mission objective, be it high speed ferries or naval craft, where speed and agility are the main priority, low weight becomes the most important design driver. 
More recent Naval ship structures are utilising carbon composite face sheets for increased weight saving, such as the Visby class corvette, Fig.1b. This has also enabled the reduction of signatures, particularly radar signatures but electromagnetic shielding can also be obtained.

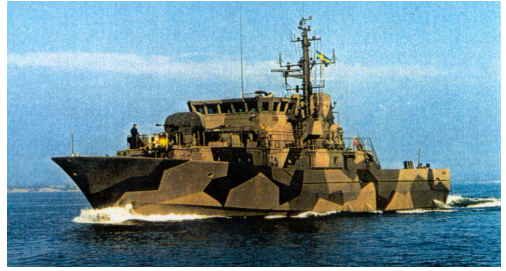

(a)

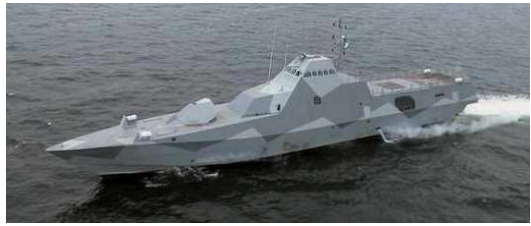

(b)

Fig.1. (a) The Landsort class mine counter measure vessel and (b) Visby class corvette. Courtesy of FMV.

Optimization methods are often used to minimize the structural weight. A minimum weight design is then sought after, while fulfilling constraints on stiffness, strength, natural frequencies, etc. The materials are then utilized highly even in normal usage. This implies that the hull structure will be designed for sea loads rather than extreme load cases such as underwater explosion and are thus designed towards more narrow limits. Another complicating factor appears in the design of more advanced composite sandwich hull structures using carbon composite face sheets. Carbon fiber laminates are much stiffer than their glass counterparts, while only giving limited increases in strength, if any. Thus, glass composite structures were mostly designed stiffness whereas carbon composite marine structures to a larger extent must be designed for strength. Localized damage generally affects the global stiffness very little whereas the strength can be reduced significantly. Strength driven components in ship hulls will be much more affected by any kind of damage, in-service damage or manufacturing defects.

Damage requires special attention both in the design process and in the operation of the structure. Historically, damage is dealt with implicitly by means of incorporating safety factors on the design loads. Damage is thus treated as any other unknown in the design process, such as material variability, fatigue, etc. However, large safety factors will induce a weight penalty and increased cost.

As summarized in [1], the possible damage types in composite sandwich structures can be divided into two categories; manufacturing flaws and in-service damage. In the former category there are those concerned with the face sheets, such as

- dry-zones and/or voids

- delaminations

- wrinkles or misalignment of fibres

- $\quad$ poor curing

For the sandwich, manufacturing flaws are;

- face/core debonds 
- $\quad$ voids and inclusions in the core

- lack of bond between core sheets

In the latter category, in-service flaws, the main worry is that of contact damage (dynamic and quasi-static), resulting in face sheet damage, delamination, skin/core debonds, core crushing or even complete penetration.

This paper summarizes the efforts by the author concerning modeling and testing of composite sandwich damage, however limited to damage related to the core material and face/core interfaces. It will be completed with a summary of a suggested damage tolerance assessment scheme as outlined in [1] and [2].

\section{Fracture of foam core materials}

Many damage tolerance models are based on fracture mechanics, i.e., the damage is assumed to have some sort of geometry so that stress field must be described as being singular. The sought after properties are fracture toughness for various modes of loading to be used as material data input in subsequent modelling of damage.

The mode I fracture toughness of polymer foam cores used in sandwich structures is described in e.g. [3]. Here, two different specimen types were used; the cracked three-point bending specimen and centre cracked specimen. The former is shown in Fig.2a.

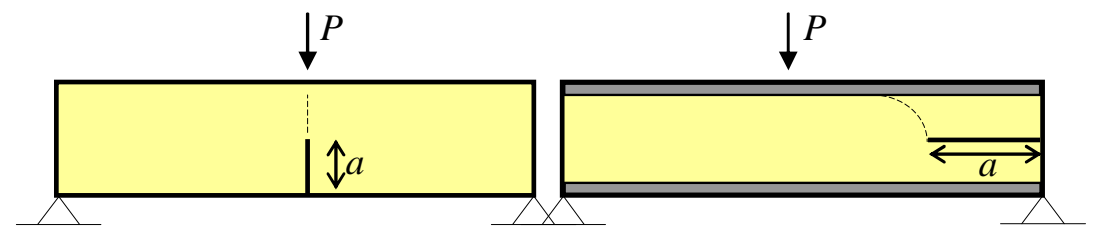

(a)

(b)

Fig.2. (a) The cracked three-point bend specimen and (b) the cracked sandwich beam specimens. Crack propagation path indicated by dashed lines.

The fracture toughness under mode II loading was later pursued [4]. The specimen type used for that purpose is shown in Fig.2b. It is a sandwich specimen where the starter crack is located in the middle of the core by placing a thin Teflon film in between two foam blocks. The crack propagation will extend in mode I, with a path indicated in Fig.2b.

The most important results from these studies are; The fracture toughness measurements are basically independent of specimen size, specimen width and crack length. The apparent mode II toughness is basically the same as the mode I fracture toughness. It was also found that the fracture toughness scales with relative density in the same way as the tensile strength. For Divinycell and Rohacell foams these relations are

$$
\sigma_{\text {ten }}=C_{1} \bar{\rho}^{1.1}, K_{I c}=C_{2} \bar{\rho}^{1.1}
$$


where $\bar{\rho}$ is the relative density of the foam (foam density divided by the density of the solid polymer).

In [5], Shipsha et al performed a comparative study of fatigue crack growth in foams using both compact tension specimens and a modified double cantilever specimen. The latter is depicted in Fig.3a. This also measure the mode I fracture toughness, but with the crack extending along the face/core interface. Actually, the measured fracture toughness is still the same as when measured using the specimens shown in Fig. 2 at least as long as the crack grows in the core, along the interface. This is usually the case unless the interface is very weak.

In order measure true mode II crack initiation and propagation one should use a specimen that simulates crack propagation along a face/core interface. The cracked sandwich beam (CSB) specimen was first proposed by Carlsson [6] and then used in [7] to measure interfacial fracture toughness. The interfacial fracture toughness is significantly higher than the mode I toughness, mainly due to the fact that the crack is forced to grow in mode II. Østergaard et al [8] recently devised a testing method to measure the interface toughness at various mode mixities, ranging from pure mode I to pure mode II. They found that the toughness remain approximately equal to the mode I fracture toughness as long as the mode II component is relatively small (mode mix down to -30 degrees) and then increases.

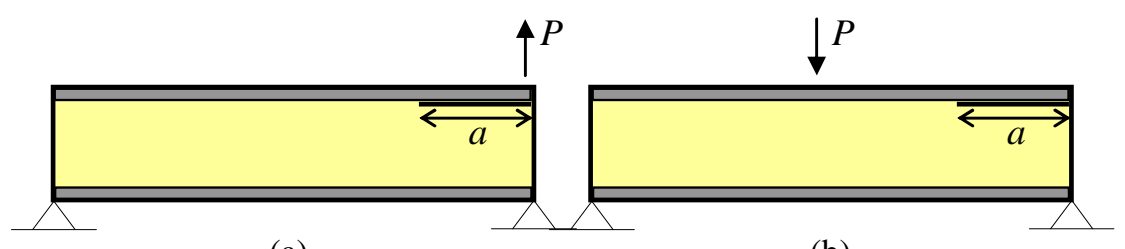

(a)

(b)

Fig.3. (a) The double modified cantilever specimen and (b) the cracked sandwich beam (CSB) specimen

In [5] and [7], tests were also performed using fatigue loading in order to measure fatigue crack propagation, both in mode I and mode II. The important findings in these papers are; The crack propagation rates in mode I is very high in structural foams and, as expected, the mode II propagation rates are lower than in mode I.

\section{Disbonds in sandwich beams}

Debonds can occur either from manufacturing or in cases from in-service impact loads. In cases where two foam core blocks are bonded together to build the required thickness, debonds can occur as depicted in Fig.4a. A more common place would be at the face/core interface, as shown in Fig.4b.

Mid-plane debonds in sandwich beams, Fig.4a, was studied in [9]. The debond was modelled analytically by means of beam theory where the energy release rate could be calculated by means closed form solutions for the total potential energy of the beam. The model was validated with FE-analyses and the correlation was very good. For validation, beams were manufactured with simulated debonds by 
means of thin Teflon films, and the beams were tested in four-point bending with the debond located between one inner and outer support, i.e., in the shear region. In order to predict the failure loads, the fracture toughness as measured using specimens of the type shown in Fig. 2 were used. The failure mode, Fig.4a, indicates that the crack propagates in mode I.

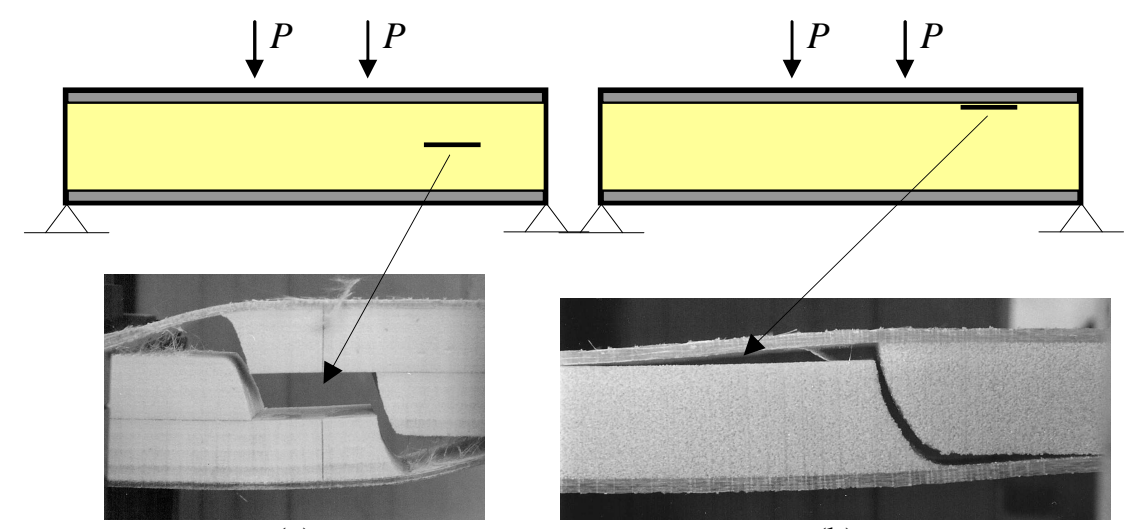

(a)

(b)

Fig.4. (a) Sandwich beam with mid-plane core debond and (b) sandwich beam with face/core interface debond. Schematic test-up above and photos of failure modes below.

In a similar study [10], interface debonds were studied. The same type of analytical model was derived, however using a simplified fracture mechanics approach. Again, the model was validated with FE-simulations with very good correlation. The failure mode prediction has to be performed for both crack fronts. The left crack front in Fig.4b exhibits the same type of loading as the CSB specimen depicted in Fig.3b. The crack "wants" to grow up towards the face sheet and is thus forced to grow along the interface and it is then assumed to start growing when the stress intensity equals the mode II interface toughness. The right crack tip, on the other hand, exhibits almost the same type of loading, but the shear stress has the opposite sign. The crack "wants" to grow in the mode I direction, which in this case is downwards into the core material, which will happen when the stress intensity equals the mode I toughness. Since the latter case predicts a lower initiation load this is what occurs, as seen in Fig.4b.

A somewhat different damage type is a flawed butt-joint, as shown in Fig.5. In the production of large sandwich structures, the core is often used as mould. The foam core is cut and bonded together to the correct shape or geometry using adhesive of putty. A large number of butt-joints are thus created, bonding the core blocks edge-to-edge. A possible manufacturing defect is when this bonding fails leaving a cavity between the core blocks. 

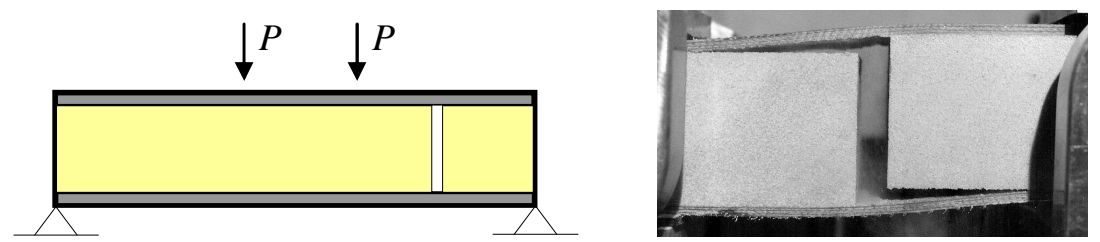

Fig.5. Sandwich beam with flawed butt-joint; schematic of test set-up and photo of failure mode.

In [11-13] this damage type was modelled and experimentally validated. The modelling was performed using FE-simulations in order to calculate so called generalised stress intensity factors. For a crack, the stress field has a so called square root singularity. The geometry of the butt-joint damage can be treated by a generalised fracture mechanics approach by computing the stress-field close to the material corner created by the flaw. The stress fields for a crack and a corner are given in Eq.(2) where $\lambda$ is the so-called strength of singularity. For a crack in homogeneous media, $\lambda$ equals 0.5 , but for a corner it takes other values, smaller than 0.5 .

$$
\sigma \propto K r^{-\lambda}
$$

The actual value of $\lambda$ was computed using a theoretical approach in [14] using potential theory. For the configurations tested it was found that $\lambda$ was 0.64 and 0.69 , implying that the strength of the singular stress field is weaker than for a sharp crack. These values were validated by using high-fidelity FE-calculations from which the stress intensity factor $K$ was also calculated. In order to make failure load predictions for beams of the kind depicted in Fig.5 one also needs the corresponding value of the fracture toughness and this has to be measured for a specimen configuration having the same singularity as the tested flawed butt-joint. This was done using modified block shear test [14]. Testing of beams with flawed butt-joints was then performed in four-point bending. The failure predictions were similar to that of the other beam damage types. The load at which the stress intensity $K$ equals the measured fracture toughness was taken as the failure load. Two different material combinations (both having glass reinforced face sheets, but with different cores), each with two different widths of the butt-joint were tested. The predictions were in all cases very good resulting in a residual strength in order of $50 \%$ of the undamaged configuration.

Both interface debonds and flawed butt-joints were also tested in fatigue load using the same type of material configurations and test set-up $[15,16]$. In a similar study [17] also beam configurations with honeycomb cores were tested in fatigue. The result of this was that the effect of the damage was the same in fatigue as for quasi-static loading, implying the SN-curve for an undamaged sandwich beam could simply be reduced with a factor to obtain the SN-curve for the damaged case, and that the factor equals the static strength reduction. 


\section{Impact damage in sandwich beams}

Perhaps one of the most common threats to structures in general is that of foreign object impact. For sandwich structures this threat is particularly important since a sandwich panel usually has thin face sheet in order to save weight. However, in the impact scenario not only the face sheet can suffer from damage but also the underlying core. Face sheet damage could be matrix cracking, delamination and fiber failures. Of particular interest is the damage occurring in the core at an impact event. Depending on the foam core type, two different failure types occur as defined in Fig.6.

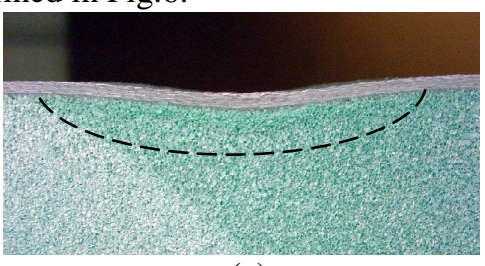

(a)

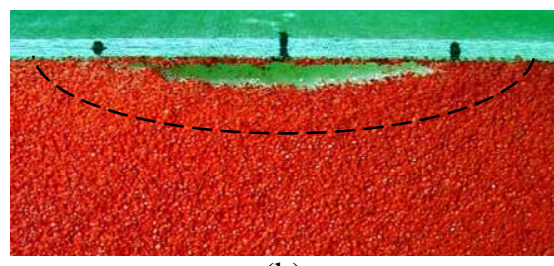

(b)

Fig.6. Impact induced core damage in sandwich beams. (a) crushed core with indent in face sheet and (b) crushed core with cavity. Dashed lines indicate extension of crushed core.

The first one implies that the core is compressed and densified, but not cracked. Upon unloading the core "holds down" the face sheet leaving a residual dent in the face sheet. The second scenario occurs for more brittle cores. At unloading after the impact event, the core fractures in tension leaving a cavity in the core resembling a crack. However, the zone of crushed core, as indicated by the dashed line in Fig.6, is larger than the cavity.

The size of the residual dent can be modelled in the quasi-static impact case $[18,19]$. This analysis model is based on the assumption that the core crushes progressively so that the strain in the core is either linear elastic or at the densification strain. The dynamic impact event creating a core cavity (Fig.6b) requires more elaborate analyses [20].

One very important aspect in the modelling of core damage is to consider the properties of the crushed foam core. The residual strength prediction models of foam core sandwich beams with impact damage used in [21-24] are all based on detailed descriptions of the crushed core zone, both in terms of geometry and the properties of the crushed core material. The crushed core properties were obtained from experiments where the core was pre-crushed in compression, allowed to relax and then tested in tension, compression and shear in various directions [22]. It was found that the as-crushed moduli and strengths dropped significantly compared to non-crushed foam and that the crushed foam became highly orthotropic.

The geometry of the impact damage requires two different loading conditions to be studied. Since the foam is crushed and has significantly reduced strength, and in some cases there is even a cavity underneath the face, shear loading is relevant, just as for an interface crack. This case was experimentally investigated in 
[22] and modelled in [23] for a configuration with a core cavity. The experimental set-up used a four-point bending configuration, as depicted in Fig.4, with the impact damage located between an inner and outer support, i.e., in the shear zone. The modelling approach used FE-analysis with a detailed description of the damage following Fig.7, where different parameters could be varied depending on damage type and impact energy [23].

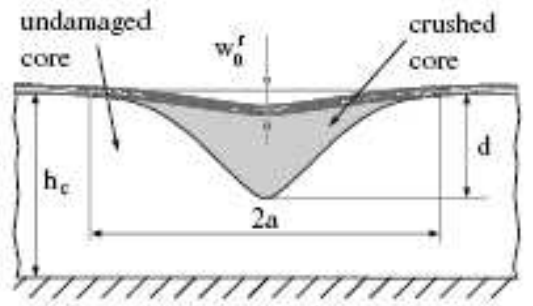

(a)

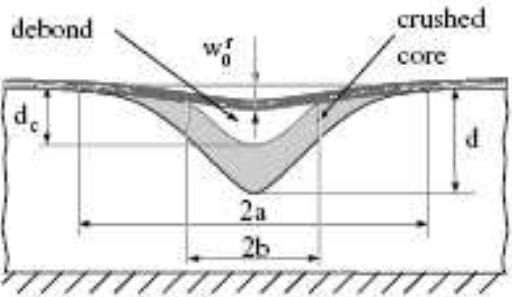

(b)

Fig.7. Idealized model with parameters for the modeling of impact damages. (a) crushed core with indent in face sheet and (b) crushed core with cavity. From [24].

As a first conservative approach, the damage was modelled assuming a crack extending from the cavity through the crushed core zone, along the interface, having the crack tip at the position where the crushed core meets the non-crushed at the face/core interface. A second, more elaborate, approach was to model this crack assuming crack bridging stresses. The second approach gave excellent comparisons with the experimental results for a range of impact energies [23]. Actually, the failure mode very much resembles that of an interface crack, as shown in Fig. $4 \mathrm{~b}$, hence the assumed crack in the crushed core zone.

The second relevant load is in-plane compression, or bending of the beam with the damage located near the compressed face sheet. Compression loading of the face sheet is particularly important due to the features of the damage having a perturbed face sheet due to the residual dent resting on a crushed core with reduced stiffness, or partly unsupported (cavity case). The same FE-model was used as in the shear, however, without the assumed crack along the interface in the crushed core zone. Buckling of the face was now sought after which was calculated using geometrically non-linear FE-analyses using Riks-Wempner arc-length control algorithm. Both bending and in-plane compression load cases were studied numerically and experimentally. The correlation between simulations and experiment were less satisfying for this case, with the numerical predictions being $20 \%$ nonconservative.

In [24] a configuration having a more ductile core was used leading to an impact as in Fig.7a, i.e., without any core cavity. The failure mode of such configurations is characterized by stable dent growth followed by a more buckling type unstable dent growth. In order to capture this properly, one still needs to accurately model both the residual face sheet dent and the geometry and material properties of the underlying crushed core.

The fatigue behaviour was also pursued in [25]. The configuration was an impact damage with a cavity as shown in Fig.7b. The loading was in shear (using 
four-point bending tests). The effect of the crushed core was evident in the tests. A fatigue crack started at the tip of the cavity and grew rapidly along the face/core interface in the crushed core until it was arrested when reaching the undamaged core, see Fig. 8 . This process took less than $20 \%$ of the fatigue life. During the rest of the fatigue life the crack remained (visually) dormant until the very last stages of the fatigue life when a crack initiated and kinked down into the core material causing failure of the specimen.

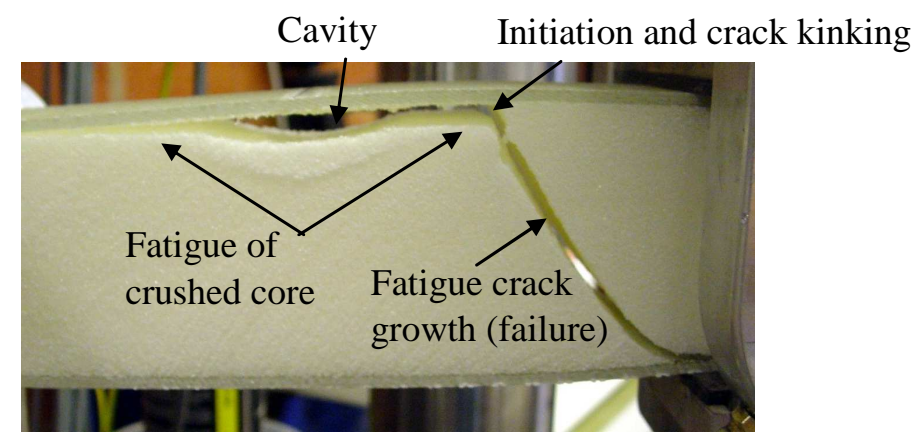

Fig.8. Fatigue failure of impact damaged sandwich beam

\section{Interface disbonds in sandwich panels}

Damage in panels is considerably less studied in the literature. This is much more relevant, however more difficult to pursue, both in terms of modelling effort and costs for experiments. Perhaps not always widely known is that the effect of damage in sandwich panels is often quite different than in beam specimens. The reason for this is mainly that damages tend to cause complete and catastrophic failure in beams, whereas damage progression often is stable in panels. This also implies that the effect of damage usually is much smaller panels than in beams.

The effect of large circular debonds in sandwich panels under hydrostatic loading were studied in [26] and [27]. The rationale for studying hydrostatic pressure stems from the design rules for hull panels which are designed for a load corresponding to a uniform pressure. A face/core debond in a sandwich panel can be positioned in different places in the panel, e.g. in the middle of the panel (Fig.9a) or across the panel boundary (Fig.9b). The panel boundaries should be interpreted as the position of underlying stiffeners on which the panel rests. 


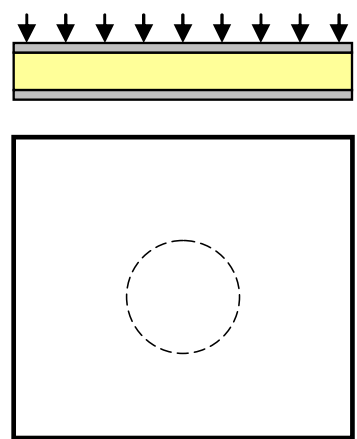

(a)
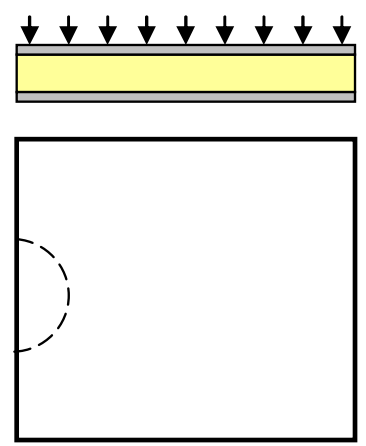

(b)

Fig.9. Schematic of face/core debond in a sandwich panel with (a) debond in the middle of the panel or (b) across the panel boundary

An attempt to analytically model the case in Fig.9a was performed in [26] by the same type of approach as for the beam case and assuming circular symmetry. This worked reasonably well but not with great accuracy. Models based on FEsimulations were also performed based again on the same approach used for beams [10]. The failure load predictions were reasonably good. Tests of large sandwich panels ( 800 by $800 \mathrm{~mm}$ ) were performed using a water bladder technique [28]. By this procedure, panels can be tested under uniform pressure loading. The debonds were simulated with a thin Teflon film placed at the face/core interface during panel manufacturing. The debonds were reasonably large, with diameters ranging from 200 to $500 \mathrm{~mm}$. Despite having so large debonds, the effect of the damage was surprisingly low and the reduction in load bearing capacity was only about $35 \%$ for the cases tested with a more ductile type core (Divinycell). The reason for this is that the transverse force in a simply supported panel subjected to uniform pressure is small in the middle and large at the boundaries. The transverse force creates core shear stresses which drives the debond crack to grow. For panels with more brittle cores (Rohacell) the reduction in load bearing capacity was larger.

In a subsequent study [27] the debond was placed at the boundary of the panel, Fig.9b, since this is the location of maximum transverse force in the panel. As expected, the FE-analysis showed that the stress intensity is much higher for this case and the experiments exhibited a much lower failure load than for a debond in the middle of the panel.

There are a few important conclusions from this work; first of all, due to the loading, contact between the face and the core along the debonded area must be considered in the numerical analysis. A second perhaps more important practical issue is that not only the size of the debond influences the severity of the damage, but also position. There is a great different between the two cases depicted in Fig.9. Furthermore, there is a difference whether the debond is located at the upper or lower interface. The studied cases has the debond in the upper interface (Fig.9) towards the side of the pressure load where the face laminate is in compression. For this case, the sign of the shear stress is such that debond crack will grow along 
the interface, similar to the test specimen in Fig.3b. The mode of failure has a high fracture toughness. If the debond is placed in the lower interface, towards the face laminate in tension, the sign of the shear stress changes. For this case, the debond crack will kink into the core material, a mode with low fracture toughness. The situations are therefore in analogy with the interface debond in a beam according to Fig. $4 \mathrm{~b}$, where one crack tip want to grow along the interface and the other kink into the core, due to the different signs of the shear stress (and thus $K_{\mathrm{II}}$ ).

Jolma et al [29] performed a similar study with debonds placed in the middle, across a boundary and at a corner. They also used a similar experimental set-up applying uniform pressure to the panels. Their analysis models were also based on $\mathrm{FE}$, but with higher accuracy and a better fracture mechanics model including the effect of mode mixity. Their failure mode predictions were in all cases excellent.

In-plane compression is perhaps an even more critical load case for sandwich panels with interface debond damage. The scenario is schematically illustrated in Fig.10. As the in-plane load is increased, the debond will start to "buckle" out. This deformation creates a non-uniform stress intensity along the debond crack front. At some load, the debond crack will start to propagate, stable or unstable, causing the panel to fail. The kinematics of the problem is geometrically nonlinear so that the stress intensity varies non-linearly with the applied load.

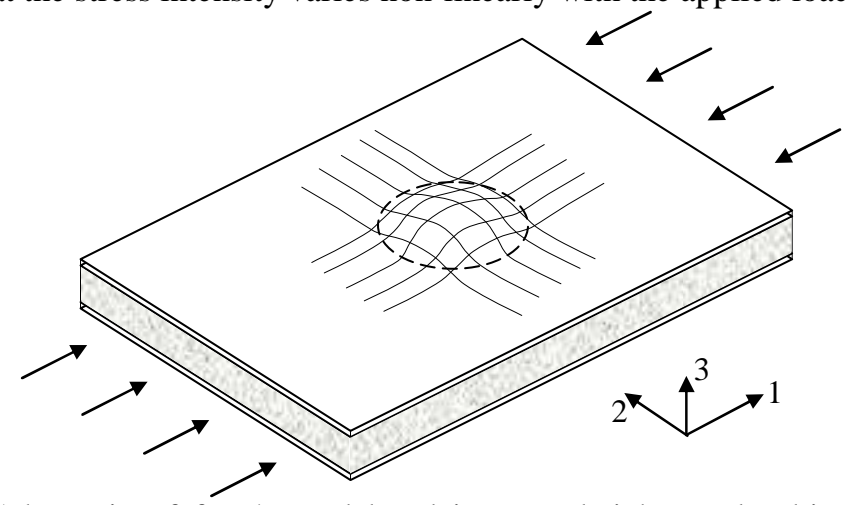

Fig.10. Schematic of face/core debond in a sandwich panel subjected to inplane compression load

This problem has been thoroughly investigated by Berggreen [30-32]. He studied sandwich panels with circular interface debonds subjected to various types of in-plane compression loading. The numerical analyses uses FE and with a highly refined mesh towards the debond crack tip circumference which enables not only the calculation of the stress intensity along the crack front, but also the mode mixity. The analysis results were combined with values for the interface fracture toughness, still regarding the current mode mixity, from which failure load predictions were made. These predictions were compared with experimental results obtained from compression tests of fairly large $(500$ by $500 \mathrm{~mm}$ ) sandwich panels with simulated interface debonds. The correlation between the numerical exercise and the experiments were overall quite good. 
There are several reports from various sources that if a debond occurs in a sandwich panel, no matter how, this disbond will be perturbed due to an internal pressure. The pressure build-up is caused by outgasing of the core material [33]. When closed cell foams are manufactured, there will be an entrapped overpressure (above atmospheric) in each cell. It is rationalized that this pressure inside individual cells of the foam core will diffuse to volumes of lower pressure, such as a debond. This means that with time, the pressure inside the debond increases to that similar to the cells. Then a blister is formed defined by a debond that due to the internal pressure "bulges" out, see Fig.10. The blister could potentially propagate in size just from the internal pressure build-up alone, but that would normally be a rather slow and stable process. The main worry is that it can propagate unstably under external loads, particularly in-plane compression that increases the deformation of the debond.

The analysis and testing of blisters is somewhat different than analyzing debonds without pressure. It involves applying a certain pressure inside the disbond, keeping the entrapped air volume in the blister while applying the external load. Numerically, this can be done by using FE-analysis. The debond must be modeled with a small gap which is filled with fluid type elements. In stage one, the pressure in the fluid is increased. This creates a volume change of the entrapped air, causing the debond to buckle out a certain amount. The next load step is to apply the external in-plane compression load while keeping the entrapped air volume constant. Due to the applied load the debond out-of-plane displacement will increase, but the pressure inside the blister will decrease due to the increased volume. In each load step the stress intensity (or energy release rate) must be evaluated along the debond crack front. All this must be performed using geometrically non-linear analysis.

In terms of experimental validation the same procedure applies. A technique to apply pressure inside a debond is the following. A sandwich panel is manufactured with a debond simulated using a thin Teflon insert. A small hole is drilled from the back side of the panel to the film insert without penetrating the face sheet. Thereafter a pressure gauge, equipped with an air inlet and a valve, is adhesively bonded over the hole on the backside. This means that the pressure can be applied in the artificial blister, the valve shut (which ascertained constant volume in the blister), and the pressure monitored during the in-plane compression loading. The signal from the pressure gauge is monitored both during the initial pressurizing stage and during the in-plane compression stage. The tests are carried out the same way as numerically analyzed. The panels are mounted in a compression test machine. First, the blister is filled with compressed air to a specified pressure. The air valve is then closed to maintain the given volume of air in the blister. The panel is then subjected to a uni-axial in-plane compressive load.

An example of such analyses and tests are shown in Fig.11. The analysis was performed using ABAQUS with the scheme described above. Fig.11 shows a plot of the pressure drop during in-plane loading. Here the pressure and load are plotted versus time (strain step) from a panel with a $50 \mathrm{~mm}$ diameter blister and an initial internal pressure of $0.2 \mathrm{MPa}$. The panel has glass/vinylester face sheets and a $50 \mathrm{~mm}$ thick $100 \mathrm{~kg} / \mathrm{m}^{3}$ foam core. The lines are taken from the experiments. The 
nominal compressive strain is taken from Digital Image Correlation (DIC) measurements and the pressure readings from the pressure gauge. It can clearly be seen that first a quick increase in pressure is obtained. After that the in-plane loading compression loading starts and the pressure starts to drop due to the increase in blister volume. The dots in Fig.11 are from the ABAQUS simulation of the same case. In order to compare, the pressure inside the blister is read from the ABAQUS output at given load increments and not for given time steps. The markers are thus created by taking the nominal compressive strain at a given increment and placing them on top of the experimentally measured points (square markers) and then plotting the blister pressure for that particular load increment (diamond markers).

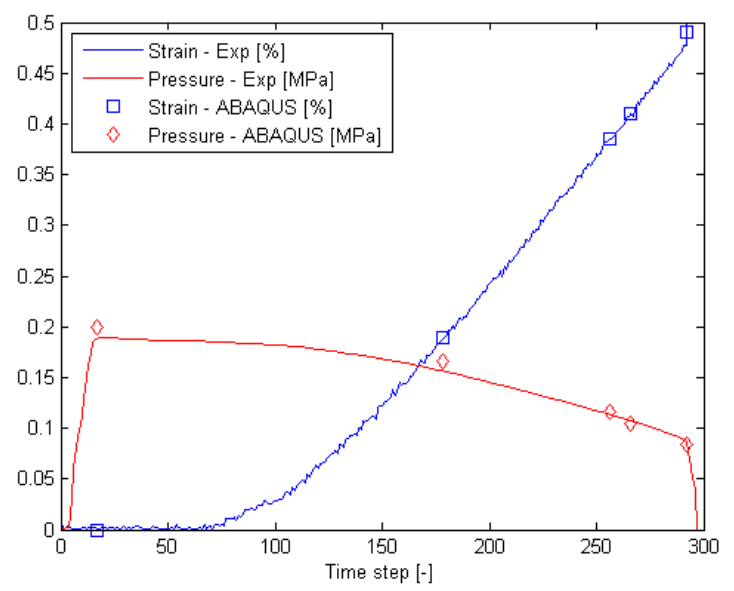

Fig.11. Blister internal pressure and applied compressive load versus time (strain step), from experiment (lines) and from ABAQUS simulation (dots).

\section{Impact damage in sandwich panels}

In [34] the residual strength of impact damaged sandwich panels was studied. The configuration was panels with fairly thin $(2.4 \mathrm{~mm})$ glass/vinylester face sheets on a $50 \mathrm{~mm}$ Rohacell foam core (50 mm thick). The panels were subjected to lowvelocity impact from a $25 \mathrm{~mm}$ diameter spherical indentor with various impact energies ranging from 10 to $60 \mathrm{~J}$. The resulting damage was of the type depicted in Figs.6b and $7 \mathrm{~b}$, i.e., core crushing, a core cavity and a remaining dent in the face sheet. For all impact energies the impact damage geometrical parameters were measured (see Fig.7b) by destructive sectioning. The face sheet laminate remained almost intact and was assumed so in the modelling.

In-plane compression tests were performed quasi-statically on panels having different impact energy damages. The panels had strain gauges mounted and the surface with the damage was also monitored using a Digital Image Correlation system set-up to measure also out-of-plane displacement by the use of two digital 
cameras. From this, the dent displacement as function of applied load could be measured.

A finite element model was constructed using the damage parameters (dent depth, crushed core geometry, etc) as input parameters as shown in Fig.12a. As for the beam studies with similar damage geometry [23], the core was assigned orthotropic properties for crushed core.

As the loading is applied the face sheet dent will start to increase, i.e., move downwards into the core. At low loads this is a fairly stable process both in the analysis and the experiments. Near some critical load, the dent displacement will increase in speed and the panels eventually failed by the face tearing off the core apparently by tensile failure. The dent profile is shown for a few load steps in Fig. 12b where this is clearly seen.

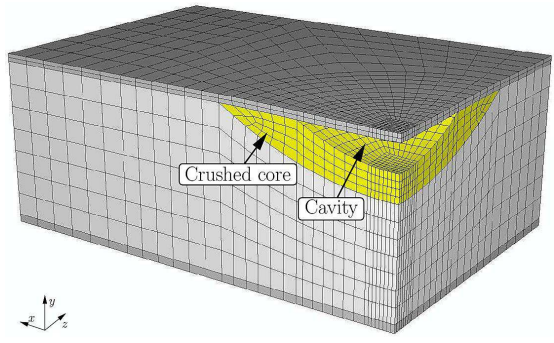

(a)

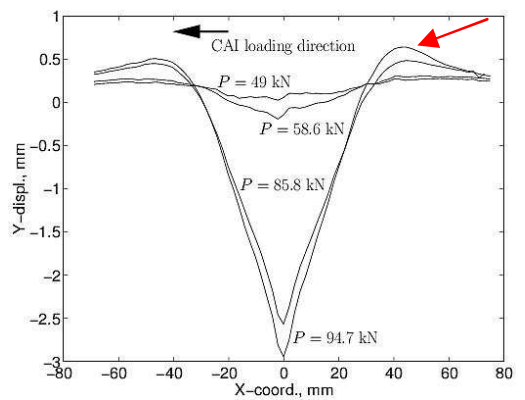

(b)

Fig.12. (a) ABAQUS-model used for sandwich panel with impact damage and (b) measured dent profile in the loading direction. Arrow in upper right corner indicates tensile core stresses. From [23].

In this case the failure load prediction was performed using two strength criteria; compressive failure of the face and tensile rupture of the core. The rationale was that either the face sheet fails in compression as compressive stresses build up in the face sheet in the middle of the dent due to the compressive load with a superimposed bending stress due to dent growth (which causes the laminate to bend). The tensile failure of the core stems from the fact that tensile stresses build up in the core above and below the dent due to the bending of the dent. This is seen in the dent profile in Fig.12b and is indicated by an arrow. Using this in the FE-model led to the prediction shown in Fig.13. The predictions thus indicate tensile core rupture, which also was verified experimentally. The failure load predictions for all the impact energies were fairly accurate. 


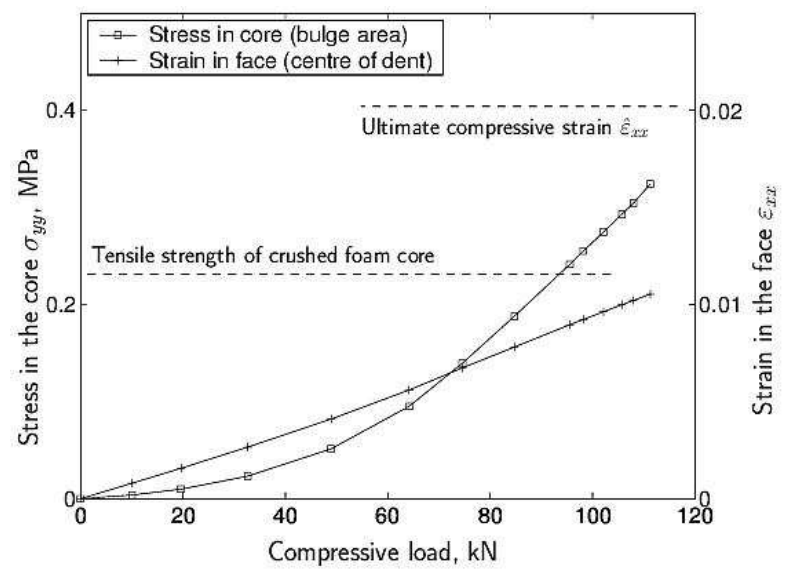

Fig.13. Tensile stress in the crushed core and compressive stress in the middle of the face sheet in the dent center. From [23].

In a similar study, reported in [2], indentation damage was studied on sandwich panels with carbon/vinylester face sheets on a $50 \mathrm{~mm}$ Divinycell core material. The damage geometry in this case has the features of that in Figs.6a and 7a, i.e. crushed core, residual dent but without any core cavity. The panels were subjected to a transverse load from $150 \mathrm{~mm}$ diameter steel indentor to varying indentation depths creating various sizes of crushed core zones and residual dents. The panels were then placed in a testing machine and subjected to an in-plane compression load until failure. Again, the panels had a number of strain gauges mounted and DIC was used to measure out-of-plane displacements in the side of the indentation damage.

An FE-model was built up and parameterized using the damage parameters indicated in Fig.7a. The model is shown in Fig.14a. The same failure criteria was used as in the previous case; compressive failure of the face sheet in the middle of the dent, and tensile rupture of the core.

As the in-plane load was applied, the dent started bending inwards towards the core. The process was fairly stable until the near critical load when the dent growth became unstable. This is shown by various measured dent profiles in Fig.14b. The DIC-measurements are shown in Fig.15 for zero-load (only showing the residual dent after indentation) and a number of loads just prior to failure. As seen herein, the dent growth is very rapid just before failure and the panel fail by compressive failure of the face sheet laminate. 


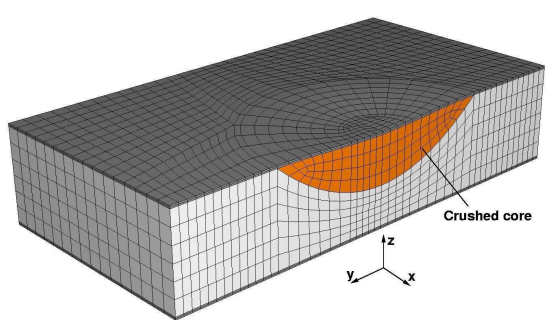

(a)

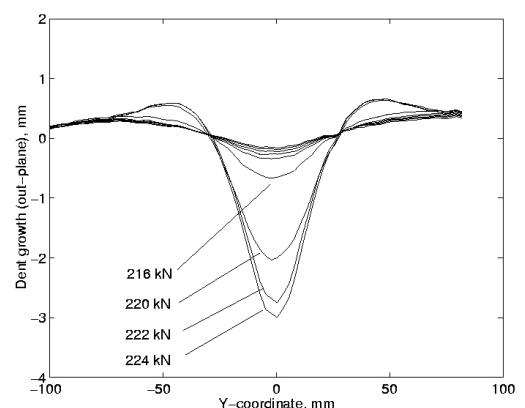

(b)

Fig.14. (a) ABAQUS-model used for sandwich panel with indentation damage and (b) measured dent profile in the loading direction. From [2].
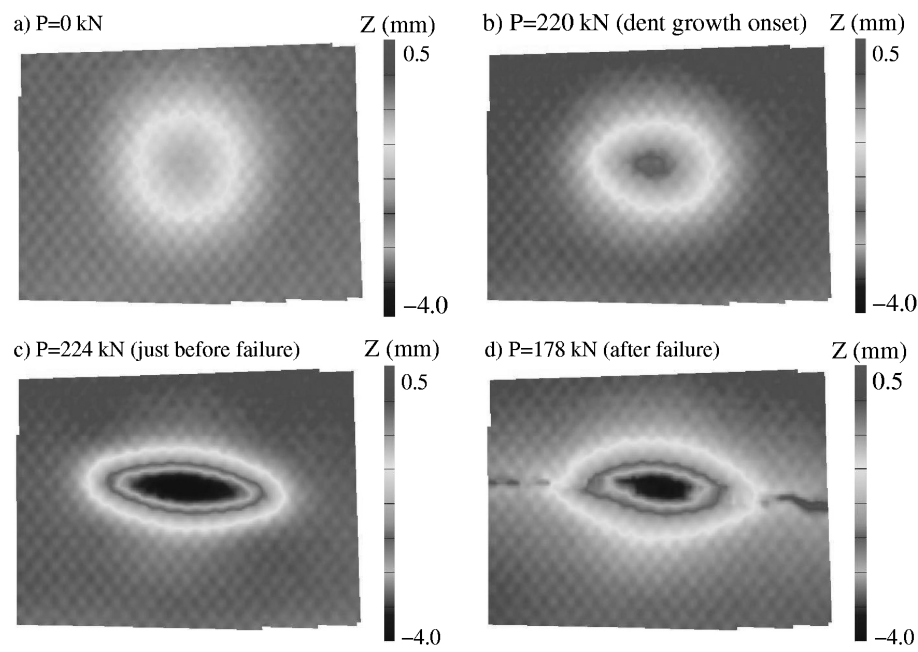

Fig.15. Digital image correlation measurements showing out-of-plane displacement. From [2].

The same type of panels was used to study two types of impact damages, one resulting from a blunt impactor and one form a sharp [2,35,36]. These are shown in Fig.16. Two different thickness' of quasi-isotropic lay-up face sheets were used with thickness $1.8 \mathrm{~mm}$ and $5.4 \mathrm{~mm}$ with a $60 \mathrm{~mm} 80 \mathrm{~kg} / \mathrm{m}^{3}$ and $50 \mathrm{~mm} 200 \mathrm{~kg} / \mathrm{m}^{3}$ foam cores, respectively. The blunt impact damage was created using a spherical head impactor with a diameter of $25 \mathrm{~mm}$. The sharp impact damage was created using a pyramid shaped impactor. The energy levels were chosen so that one level created a barely visible damage (BVID) and a second higher level gave clearly visible damage (VID). The blunt impact damage consisted of a very small dent with some minor surface fiber breakage in the VID case. The underlying core 
showed a small zone of crushing, but apart from that there was no other visible damage indicated. A cross-section of the impact zone is shown in Fig.16a. However, ultrasonic C-scan investigations revealed significant overlapping delaminations with a diameter $2 R=15-30$ [35]. The sharp type impact created a slit in the face sheet, as shown in Fig. 16(b). The projected length of this slit was in the order of $2 a=15$ to $25 \mathrm{~mm}$, with the higher value for the higher impact energy.

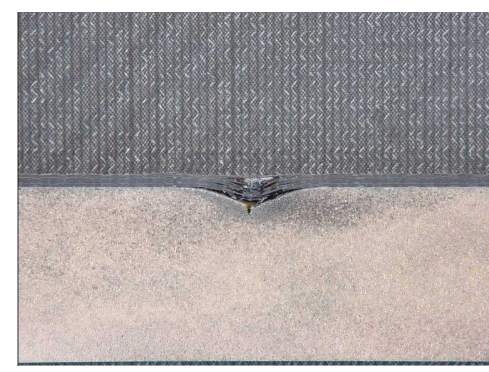

(a)

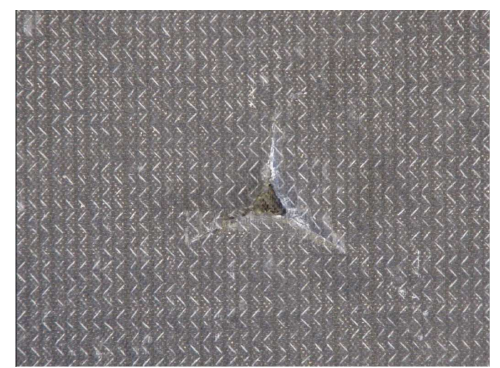

(b)

Fig.16. Damage types occurring from (a) blunt object impact and (b) sharp object impact. From [2].

The panels were then tested in in-plane compression while using a DIC-system to measure the in-plane strain field. Some tests were interrupted just prior to failure [35], and small pieces of the laminate close to the impact point was cut out, ground and polished stepwise in the thickness direction in order to identify location of failure. Even though the panel had not yet failed, this fractographic investigation revealed numerous local compressive failures manifested by kink bands (micro-buckling failures). These appeared mainly in the 0-degree plies, appearing to start at the edge of the impact damage extending outwards to the panel edges. Kink-band lengths of over $20 \mathrm{~mm}$ were found.

The DIC-measurements revealed similar evidence. The surface strain mapping revealed clear indications of stable micro-buckling growth before final failure with extensions similar to what was found through fractography. Two such DSP strain map plots are shown in Fig.17 for a thin panel with a BVID (30J) blunt impact damage. The first (Fig.17a) shows the axial strain at $120 \mathrm{kN}$ load, which corresponds to approximately half the failure load. The second strain map (Fig.17b) shows the axial strain just prior to failure (at $216 \mathrm{kN}$, or approximately $200 \mathrm{MPa}$ nominal stress). The localized strain (dark area) corresponds to an axial strain of $1 \%$. As seen, strains localize near the damage and damage progresses in a stable manner during the loading of the panel. No visible damage progression could be seen on the surface of the panel until complete fracture. 


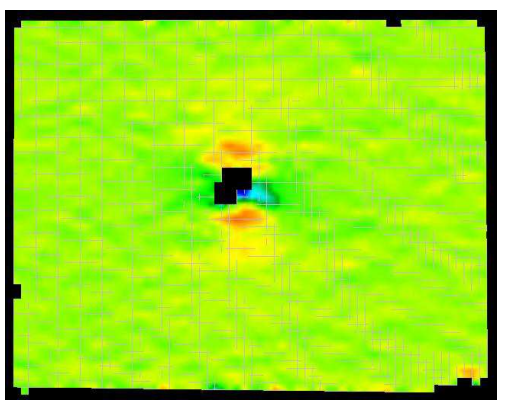

(a)

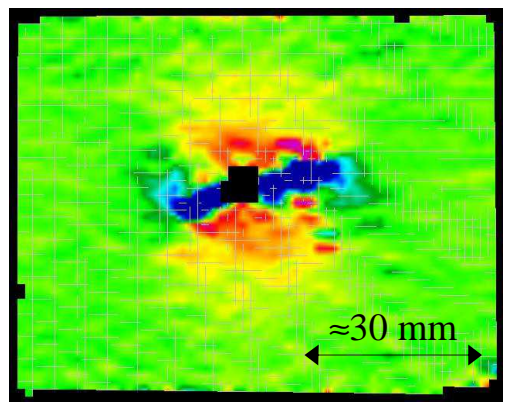

(b)

Fig.17. Axial strain map (vertical = load direction) (a) at $120 \mathrm{KN}$ load and (b) just before failure (at $216 \mathrm{kN}$ ) for a thin panel with a blunt impact damage. The dark area corresponds to compressive strains of $1 \%$. From [2].

For the predictions, the code Composite Compressive Strength Modeller CCSM [37], which implements progressive micro-buckling analysis, was used for the blunt impact cases by assuming an equivalent hole representing the damage. The same code was again used for the sharp impact tests, but then an equivalent crack model was used, since a clear crack was produced by the impact. The crack length used in the model was the projected length of the slit, perpendicular to the load direction.

In order to find the necessary input data for the CCSM model, un-notched compression tests were performed on the laminates using the Wyoming modified IITRI test set-up. To find the fracture energy, small panel specimens (150 by 150 $\mathrm{mm}$ ) were manufactured with central holes cut. These were tested in compression from which the fracture energy can be deducted by the use of CCSM. The predictions obtained from this gave excellent results in terms of failure loads and reasonable accuracy in the kink band lengths. In a sub-sequent paper, Edgren et at [38] performed a similar but more refined analysis of the same experiments.

\section{Damage tolerance scheme for Naval sandwich structures}

From a damage tolerance perspective there are several important aspects to consider. One cannot guarantee that damage or defects can be detected, both since NDI of sandwich structures is difficult due to their multi-material constitution and that ship structures are indeed very large. From a practical point of view one must therefore consider only damages that are barely visible or clearly visible. The question often asked is - "Does all damage have to be repaired?" and "Is this damage critical for the integrity of the structure?". Decisions must be taken on corrective measures relatively quickly once damage has been found. Large damages will obviously be found and often requires immediate repair, either for structural reasons or for functional reasons (water tightness, etc.). The problem addressed herein concerns barely visible impact damage (BVID) and visible impact damage (VID) with the aim of finding how much such damage will affect the load 
carrying capacity of a panel or component in the ship. Large damage is thus addressed but the focus is on damage that may exist without being detected, or damage that is small enough not requiring repair for functional reasons. Such damage may, or may not, require repair depending on its size, its type and the type of loading at its location.

In this paper, only damage related to the core and face/core interface are investigated. From a practical viewpoint all possible threats and damage types must be included in order to provide a comprehensive damage assessment system. To do this one needs not only the information on the localised strength reduction due to damage for individual panels but a more elaborate scheme for evaluating the influence of the damage on the structural performance and functionality of the ship as a whole. In the SaNDI project such a scheme was developed that is fairly generic in terms of damage types and ship structures, although the designs and design requirements may vary significantly. The damage assessment procedure for cases where strength is the main consideration is basically as follows:

- Estimate the strength reduction caused by the damage or defect.

- Determine the allowable strength reduction based on the original design assumptions, operational envelope, etc.

- Compare these. If the residual strength is smaller than the allowable value, consider the possibilities for restricting the operational envelope and/or accepting a lower safety factor until repair can be effected.

- If this is not sufficient, carry out an emergency repair or take other emergency measures as necessary.

To do this, one may need to consider the damage in the local, panel and global (ship) contexts, as illustrated in Fig.18. The scheme as a whole is shown schematically in Fig. 19.

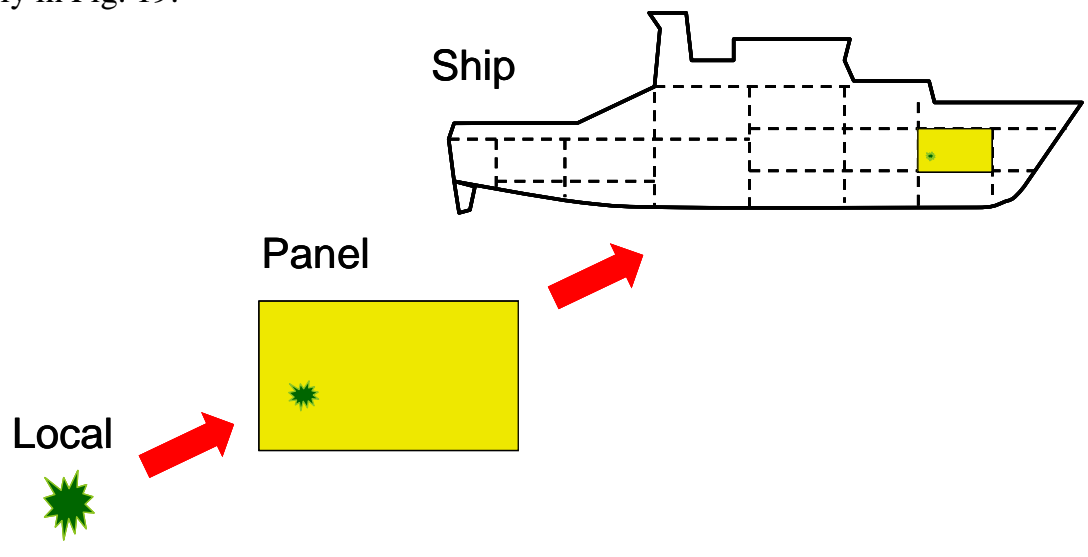

Fig. 18. Damage illustrated in local, panel and global (ship) contexts. From [1, 2]. 


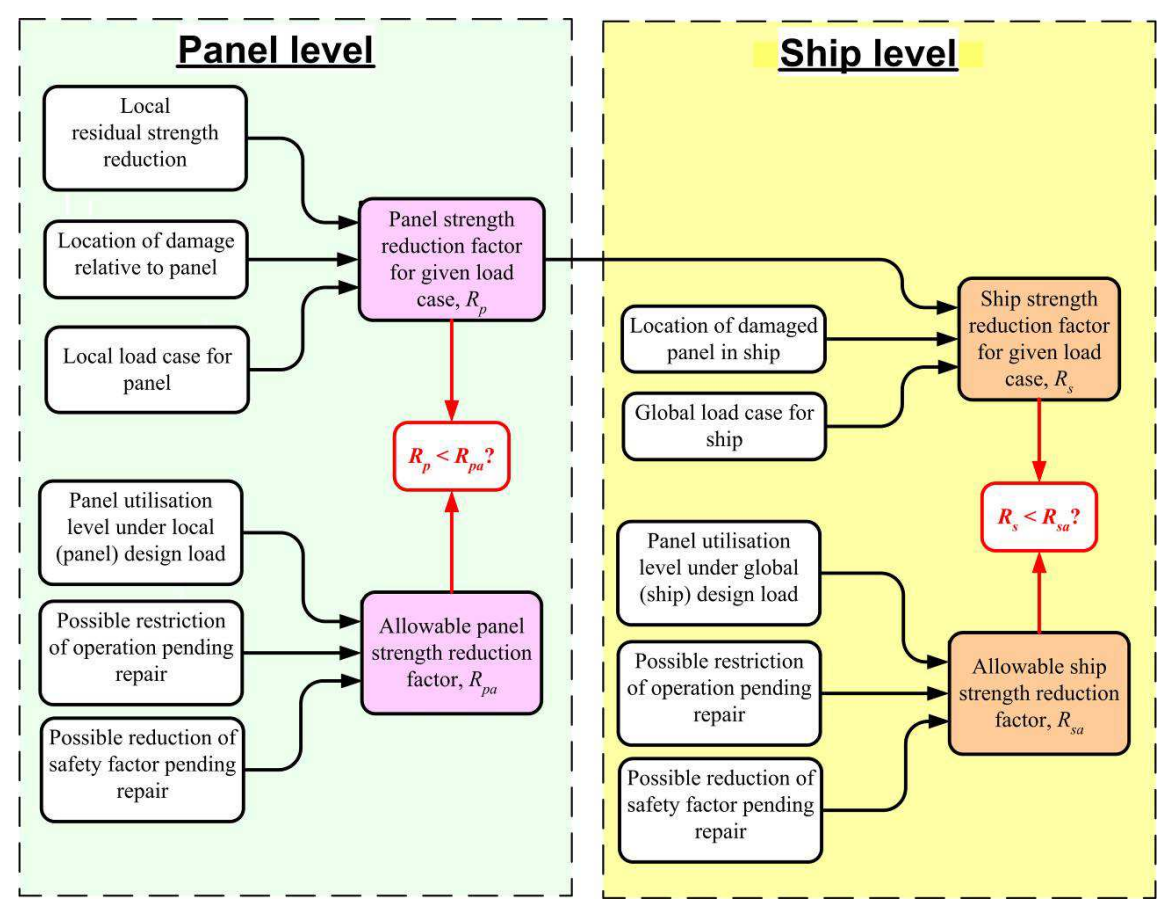

Fig.19. Residual strength approach for local panel strength and global ship strength. From [1, 2].

For damage confined to a single panel, we first consider the influence of the damage on the affected panel or component by estimating a strength reduction factor for the panel, $R_{p}$. This is then compared with the allowable strength reduction for the panel, $R_{p a}$. If the panel suffers from a reduced load bearing capacity, we need further to consider any implications of this at the global (ship) level and estimate any reductions in the global ship strength. For this purpose it is possible to calculate a ship strength reduction factor $R_{s}$, which again must be compared with some allowable global strength reduction factor, $R_{s a}$.

A division is made considering the level of damage, ranging from small local damage confined to a small part of one panel (level 1) to extensive damage affecting larger areas of two or more panels (level 4). For level 1 damage, which applies to the damage cases considered herein, the size of the damage is small compared to the panel size so that the stress distribution within the panel is unaffected by the presence of the damage (no stiffness changes). The assessment is then performed as follows:

- Determination of the local strength reduction factor $R_{l}$ that quantifies the reduction in far-field stress or strain at failure. The value of $R_{l}$ 
should preferably already been calculated a priori, and recorded in tables or graphs.

- Determination of a sensitivity factor $S_{p}$ that accounts for the location of the damage in relation to the stress field in the panel for the real loading case.

- Combination of these factors to give the panel strength reduction factor $R_{p}=R_{l} S_{p}$ or 1.0 , whichever is smaller.

The sensitivity factor $S_{p}$, which is more correctly referred to as the local location and load case factor, also provides a means of seeing immediately what are most likely to be the critical locations of damage on a panel. It is defined as follows:

\begin{tabular}{|l|l|}
\hline$S_{p}=\frac{P_{a l i}}{P_{a p i}}$ & (3) \\
\hline
\end{tabular}

where $P_{a l i}$ is the value of load that causes the critical stress or strain component at the damage location to reach its maximum allowable value ignoring the damage, and $P_{a p i}$ is the maximum allowable value of load on the intact panel. For face sheet damage, the critical stress or strain component is usually assumed to be the in-plane compressive stress or strain at the point in question. $S_{p}$ can never be smaller than unity.

As an example, suppose a panel under uniform lateral pressure is designed so that it reaches the allowable limit for core shear stresses before the allowable stresses for face laminate failure. (This will often be the case for panels designed to classification rules, for example, if the minimum thickness requirements result in an increase of laminate thickness relative to the basic strength requirements.) Then $P_{a p i}$ is the pressure load at which the allowable core shear stress is reached. Suppose that there is a local impact damage that has reduced the local in-plane compressive strength of the face laminate so this has to be checked at the damage location. Then $P_{a l i}$ is the pressure applied to the panel at which the allowable compressive stress (or strain) in the face laminate at that location reaches its maximum allowable value, also calculated for the intact panel. Note that all these quantities are calculated for the intact panel. This means that maps of $S_{p}$ values can readily be drawn for panels with given dimensions and lay-ups for simple load configurations like uniform lateral pressure.

There is normally no need to consider an allowable strength reduction if $R_{p}=1$. If $R_{p}<1$ the possibility of accepting a reduction of panel strength must be considered unless the damage can be fully repaired immediately. If $R_{p}<1$, the global strength reduction $R_{s}$ must also be evaluated. For Level 1 damage, and also for some cases of slightly larger damage to a single panel defined as Level 2 damage, it is possible to neglect redistribution of stresses between panels (and other elements) in the structure when estimating the influence of the damage on the global strength. This enables $R_{s}$ to be found from $R_{p}$ by a procedure analogous to that for deriving $R_{p}$ from $R_{l}$. For larger damage cases (Level 3 or 4 ), alternative procedures must be used as discussed in [1]. 
In some cases it is not possible to accept any reduction in the global or local strength of the structure. However, not all parts of the structure of a ship are highly stressed. In many cases a given panel may be exposed to a maximum loading that is lower than the allowable value because the design gave more than the minimum required reserve of strength, i.e. there is a lower utilization of the panel than the maximum that is allowed. This is often due to the fact that a limited number of standard sandwich lay-ups are generally used in any given vessel. In such cases it will normally be acceptable to reduce the panel strength by an amount that reflects this extra reserve of strength in the intact structure. The same may apply at the global ship level if the ship has been generally over-designed against the global loads (e.g. because the local load requirements were more severe). These aspects are dealt with here by using the utilization factors $U_{p}$ and $U_{s}$. The panel utilization factor, $U_{p}$, is defined as the ratio between the ratio of the maximum load applied to the damaged panel under extreme load to the maximum allowable value of that load for the undamaged panel. The utilization factors $U_{s}$ is defined the same way but for the global load acting on the whole structure [1,2].

Normally the allowable strength reductions for panel and global strength will be set equal to the respective utilization factors:

$$
R_{p a}=U_{p} \text { and } R_{s a}=U_{s}
$$

There are two main additional considerations that may make a strength reduction acceptable:

- Reducing the loads (relative to the original design) by restricting the operation in some way.

- Accepting a reduced factor of safety in the interim period until a repair is effected.

The first of these leads in effect to a lowering of the utilization level in that the extreme design loads are now decreased. The second leads to a lowering of the utilization level in that the allowable loads are increased.

Note that the procedure described so far deals only with strength considerations. Considerations of functionality (which may include that of stiffness as embodied in deflection criteria) must be made separately. Functionality requirements, such as

- watertightness

- weathertightness

- smoke-tightness

- $\quad$ fire division effectiveness

- $\quad$ equipment functioning (machinery, instrumentation)

- $\quad$ signatures and shielding

- other functionality

may override any repair decisions based on load carrying capacity. Thus, although a damage does not require repair from a structural point of view, functionality may still require repair.

Finally, decisions about corrective measures have to be made on the basis of comparing the strength reductions $R_{p}$ and $R_{s}$ with their allowables $R_{p a}$ and $R_{s a}$. 
Fig.20 shows an example of a flow-chart for such a decision-making process, taking account of only the strength reduction aspects.

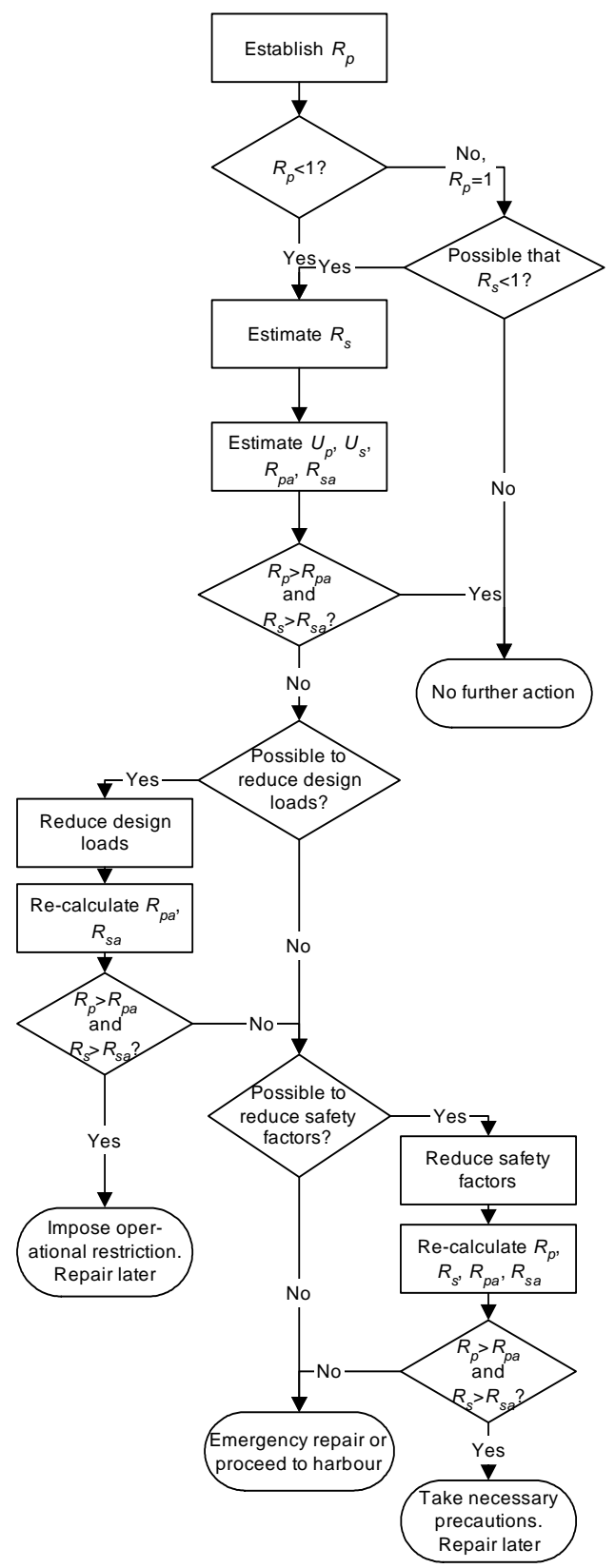

Fig. 20. Schematic illustration of assessment process. From [2]. 


\section{Acknowledgements}

The work summarised in this chapter has been ongoing since the 80'ies with various support. Thanks are due to The Swedish Defence Materiel Administration and Mr. Anders Lönnö for funding parts of this. The Nordic Industrial Fund (NI) provided support for a long time in the 1990'ies. A lot of the more recent work was performed within the WEAG-project saNDI in collaboration with Prof. Brian Hayman at DNV. During most of the past 10 years invaluable support has been provided from the Structural Mechanics Programme of ONR through programme officer Dr. Y. Rajapakse.

\section{References}

1. Hayman B (2007) Approaches to damage assessment and damage tolerance for FRP sandwich structures. J Sand Struct Mater 9: 571-596

2. Zenkert D, Bull P, Shipsha A, Hayman B (2005) Damage tolerance assessment of composite sandwich panels with localised damage. Comp Sci Tech 65: 2597-2611

3. Zenkert D, Bäcklund J (1989) PVC Sandwich core materials: Mode I fracture toughness. Comp Sci Tech 34: 225-242

4. Zenkert D,(1989) PVC Sandwich core materials: fracture behaviour under mode II and mixed mode loading. Mat Sci Eng A108: 233-240

5. Shipsha A, Burman M, Zenkert D (2000) On mode I fatigue crack growth in foam core materials for sandwich structures. J Sand Struct 2: 103-116

6. Carlson LA (1991) On the design of the cracked sandwich beam (CSB) specimen. J Reinforced Plastics 10: 434-444

7. Shipsha A, Burman M, Zenkert D (1999) Interfacial fatigue crack growth in foam core sandwich structures. Fatigue \& Fracture of Engineering Materials \& Structures 22: 123-131

8. Østergaard RC, Sørensen BF, Brøndsted P (2007) Measurement of interface fracture toughness of sandwich structures under mixed mode loadings. Sandwich Struct Mater 9: 445-466

9. Zenkert D (1990) Strength of sandwich beams with mid-plane debondings in the core. Comp Struct 15: 279-299

10. Zenkert D (1991) Strength of sandwich beams with interface debondings. Comp Struct 17: 331-350

11. Zenkert D, Groth HL (1989) The influence of flawed butt-joints in foam core sandwich beams. In: Olsson KA, Reichard RP (eds) First Int Conf on Sandwich Constructions, EMAS Ltd., UK, pp 363-381

12. Groth HL, Zenkert D (1990) Fracture of Defect Foam Core Sandwich Beams. ASTM J Testing \& Eval 18(6): 390-395

13. Zenkert D (1992) Effect of manufacturing-induced flaws on the strength of foam core sandwich beams. In: Masters JE (Ed) Damage Detection in Composite Materials, ASTM STP 1128, ASTM, Philadelphia, pp 137-151 
14. Zenkert D, Schubert O, Burman M (1997) Fracture initiation in foam core sandwich structures due to singular stresses at corners of flawed butt-joints. Mech Comp Mater Struct 4(1): 1-21

15. Burman M, Zenkert D (1997) Fatigue of foam core sandwich beams, Part I: Undamaged Specimens. Int J Fatigue 19(7): 551-561

16. Burman M, Zenkert D (1997) Fatigue of foam core sandwich beams, Part II: Effect of Initial Damages. Int J Fatigue 19(7): 563-578

17. Burman M, Zenkert D (2000) Fatigue of undamaged and damaged honeycomb sandwich. J Sand Struct 2: 50-74

18. Zenkert D, Shipsha A, Persson K (2004) Static indentation and unloading response of sandwich beam. Comp B 35(6-8): 511-522

19. Rizov V, Shipsha A, Zenkert D (2005) Indentation study of foam core sandwich panels. Comp Struct 69: 95-102

20. Koissin V, Shipsha A (2008) Residual dent in locally loaded foam core sandwich structures - Analysis and use for NDI. Comp Sci Tech 68: 57-74

21. Hallström S, Shipsha A, Zenkert D (2000) Failure of impact damaged foam core sandwich beams. In: Rajapakse YDS (Ed) ASME ICEME'2000, ASME AERO/AMD AD-Vol. 62/AMD Vol. 245, pp 11-19

22. Shipsha A, Hallström S, Zenkert D (2003) Failure mechanisms and modelling of impact damage in sandwich beams - A 2D approach: Part I - Experimental Investigation. J Sandwich Struct Mater 5(1): 7-31

23. Shipsha A, Hallström S, Zenkert D (2003) Failure mechanisms and modelling of impact damage in sandwich beams - A 2D approach: Part II - Analysis and Modelling. J Sandwich Struct Mater 5(1): pp 33-51

24. Koissin V, Skortsov V, Shipsha A, (2007) Stability of the face layer of sandwich beams with sub-interface damage in the foam core. Comp Struct 78: 507-518

25. Shipsha A, Zenkert D (2003) Fatigue behaviour of foam core sandwich beams with sub-interface impact damage. J Sand Struct Mater 5(1): 147-160

26. Zenkert D, Falk F (1991) Interface Debondings in foam core sandwich beams and panels, In: Springer G, Tsai S (Eds) Proc Int Conf Comp Mater (ICCM/VIII), Publ by SAMPE, 3-H

27. Falk F (1992) Strength of foam-core sandwich panels with face-to-core debonds. In: Weissman-Berman D, Olsson KA (Eds) Prof. $2^{\text {nd }}$ Int Conf Sandwich Constructions, EMAS Ltd., UK, pp 645-663

28. Wennhage P, Zenkert D (1998) Testing of sandwich panels under uniform pressure. J Testing \& Evaluation 26(2): 101-108

29. Jolma P, Segercrantz S, Berggreen C (2007) Ultimate failure of debond damaged sandwich panels loaded with lateral pressure - An experimental and fracture mechanics tudy", J Sand Struct Mater 9: 167-196

30. Berggreen, C. (2004). Damage tolerance of debonded sandwich structures, $\mathrm{PhD}$ Thesis, Department of Mechanical Engineering, Technical University of Denmark.

31. Nokkentved A, Lundsgaard-Larsen C, Berggreen C (2005) Non-uniform compressive strength of debonded sandwich panels - I. Experimental investigation. J Sand Struct Mater 7(6): 461-482 
32. Berggreen C, Simonsen BC (2005) Non-uniform compressive strength of debonded sandwich panels - II. Fracture mechanics investigation. J Sand Struct Mater 7(6): 461-482

33. Causes, mechanisms and preventive actions to avoid blistering, Technical Bulletin, DIAB AB, www.diabgroup.com

34. Shipsha A, Zenkert D (2005) Compression-after-impact strength of sandwich panels with core crushing damage. App Comp Mater 12(3-4): 149-164.

35. Edgren F. Asp L, Bull P (2004) Compressive failure of impacted NCF composite sandwich panels - characterisation of failure process. J Comp Mater 38(6): 495-514

36. Bull P, Edgren F (2004) Compressive strength after impact of CFRP-foam core sandwich panels. Comp B 35: 535-541

37. Sutcliffe M, Xin A, Fleck NA, Curtis P (1999) Composite compressive strength modeller, Engineering Department, Cambridge University, UK

38. Edgren F, Soutis C, Asp L (2008) Damage tolerance analysis of NCF composite sandwich panels. Comp Sci Tech, 68(13): 2635-2645 